\title{
TÉCNICAS LMI PARA ANÁLISE DE SISTEMAS COM RESTRIÇÕES ALGÉBRICAS NO ESTADO
}

\author{
Karina A. Barbosa* \\ karinab@lcmi.ufsc.br
}

\author{
Alexandre Trofino ${ }^{\dagger}$ \\ trofino@lcmi.ufsc.br
}

* Departamento de Automação e Sistemas

Universidade Federal de Santa Catarina, SC, Brasil

\section{ABSTRACT}

LMI techniques for the analysis of descriptor systems -In this paper we present LMI techniques for the quadratic stability of uncertain continuous and discrete-time descriptor systems. Connections between the quadratic stability of systems with polytopic and norm bounded LFT uncertainties are also presented. Stability conditions are presented for the "primal" and "dual" systems.

KEYWORDS: LMI, robustness, descriptor systems, quadratic stability.

\section{RESUMO}

Neste artigo apresentam-se técnicas para a análise da estabilidade de sistemas com restrições algébricas no tempo contínuo e discreto com uma abordagem por LMIs. Apresenta-se também uma conexão entre a estabilidade quadrática de sistemas com incertezas do tipo politópica e LFT. As condições de estabilidade são apresentadas tanto para o sistema "primal"como para o "dual".

PALAVRAS CHAVES: LMI, robustez, sistema descritor, estabilidade quadrática.

Artigo submetido em $20 / 12 / 00$

1a. Revisão em 04/09/01

Aceito sob recomendação do Ed. Cons. Prof. Dr. Liu Hsu

\section{INTRODUÇÃO}

Sistemas com restrições algébricas, conhecidos também como sistemas descritores ou sistemas singulares, são representados por

$$
E \dot{\zeta}=A \zeta
$$

onde $\zeta \in R^{n_{\zeta}}$, representa o vetor de estados do sistema e $A \in R^{n_{\zeta} \times n_{\zeta}}$ e $E \in R^{n_{\zeta} \times n_{\zeta}}$ são as matrizes de dinâmica do sistema, com o posto $(E)<n_{\zeta}$, caracterizando a singularidade do sistema.

Estuda-se aqui o problema de análise da estabilidade para o sistema (1), já na forma algébrico-diferencial desacoplada, i.e. uma equação diferencial do tipo $\dot{x}=A_{1} x+A_{2} z$ com uma restrição algébrica do tipo $0=A_{3} x+A_{4} z$. Freqüentemente, sistemas descritores não aparecem na forma de um conjunto desacoplado de equações algébrico-diferenciais. No entanto, este desacoplamento pode ser obtido através de trocas de coordenadas como feito em Dai (1989); ou, parcialmente, por transformações ortogonais, como propôs Varga (1995).

A análise da estabilidade de sistemas com restrições algébricas na forma algébrico-diferencial tem um importante papel no estudo da teoria de sistemas singulares e suas aplicações. Normalmente, esta análise é feita através da eliminação da variável algébrica, transformando o sistema algébrico-diferencial em um outro puramente diferencial de menor dimensão (Cobb, 1984; Varga, 1995). Com o sistema na forma diferencial é possível aplicar técnicas de análise já conhecidas. Apesar de simples, 
essa abordagem pode implicar em diversos problemas. Um deles é a dificuldade em considerar incertezas no modelo, já que a eliminação da variável algébrica envolve inversões de matrizes com elementos incertos. Outro problema é a perda da esparsidade das matrizes que, em alguns casos, quando a dimensão do problema é grande, pode acarretar dificuldades computacionais consideráveis.

Neste artigo é proposta uma forma alternativa de estudar a estabilidade de sistemas com restrições algébricas. De uma maneira semelhante ao resultado dado em Takaba et al. (1995), explora-se as propriedades estruturais das equações do sistema original sem a necessidade da eliminação da variável algébrica, facilitando assim, o estudo de sistemas com parâmetros incertos. Considera-se então o problema de Estabilidade Quadrática, para sistemas com incertezas descritas na forma politópica de tempo contínuo e discreto. As condições para resolução dos problemas de análise e síntese serão expressas como desigualdades matriciais lineares (LMIs-do inglês Linear Matrix Inequalities), e que são tratáveis numericamente de maneira eficiente.

O presente artigo tem a seguinte estrutura. A seção 2 apresenta a definição matemática de sistemas incertos com restrições algébricas, bem como alguns resultados auxiliares úteis para o desenvolvimento deste trabalho. Na seção 3 considera-se o problema de análise para sistemas no tempo contínuo. O caso discreto pode ser visto na seção 4. Uma relação entre as abordagens politópica e LFT para representação de incertezas se encontra na seção 5. Na seção 6, exemplos numéricos são apresentados e por fim na seção 7 se encontram as principais conclusões deste trabalho.

A seguinte notação será utilizada neste artigo.

$$
\text { Co }\left[\tilde{J}_{i}\right]_{i=1}^{q} \triangleq\left\{\Psi: \Psi=\sum_{i=1}^{q} \alpha_{i} \tilde{J}_{i}, \forall \alpha_{i} \geq 0: \sum_{i=1}^{q} \alpha_{i}=1\right\}
$$

representa o politopo (convex hull) de um conjunto de vértices $\tilde{J}_{i}$ conhecidos. As desigualdades matriciais do tipo $P>0(P \geq 0)$ indicam que a matriz $P$ é positiva definida (positiva semi-definida), isto é, simétrica com todos os autovalores positivos (positivos ou nulos). Em uma matriz simétrica, será usado o símbolo $*$ para representar os elementos que podem ser deduzidos por simetria.

\section{DESCRIÇÃO DO PROBLEMA}

Considere o sistema (1) na forma algébrico-diferencial desacoplada dada por:

$$
\left\{\begin{array}{l}
\dot{x}=J_{1} x+J_{2} z \\
0=J_{3} x+J_{4} z
\end{array}, \quad J=\left[\begin{array}{ll}
J_{1} & J_{2} \\
J_{3} & J_{4}
\end{array}\right]\right.
$$

onde $x \in R^{n}$ representa os estados e $z \in R^{l}$ representa as variáveis algébricas do sistema, isto é, algebricamente dependente do estado e $J$ é a matriz jacobiana do sistema. Admite-se ainda que o sistema (2) é incerto e

$$
J \in C o\left[\tilde{J}_{i}\right]_{i=1}^{q} \quad \tilde{J}_{i}=\left[\begin{array}{ll}
J_{1 i} & J_{2 i} \\
J_{3 i} & J_{4 i}
\end{array}\right]
$$

onde $C o\left[\tilde{J}_{i}\right]_{i=1}^{q}$ é um politopo convexo de vértices $\tilde{J}_{i}$ conhecidos . No restante do artigo refere-se a este sistema como sistema algébrico-diferencial (sem mencionar que é desacoplado).

Para a plena compreensão dos resultados propostos neste artigo faz-se necessário algumas definições e resultados preliminares que serão apresentados a seguir na forma de lemas.

Definição 1 (Sistema Admissível) Um sistema com restrições algébricas é admissível se for regular e livre de modos impulsivos.

Pelo resultado apresentado em Verghese et al. (1981), tem-se que um sistema na forma (2) será admissível se e somente se a matriz $J_{4}$ for inversível.

Definição 2 (Estabilidade Quadrática) Considere o sistema (2),(3) e suponha que ele seja admissível. Então este sistema é quadraticamente estável se existe uma função $v(x)=x^{\prime} P x>0, \forall x$ tal que sua derivada temporal para as trajetórias do sistema (2), (3) satisfaça:

$$
\left\{\begin{array}{l}
\dot{v}(x)=\left[\begin{array}{l}
x \\
z
\end{array}\right]^{\prime}\left[\begin{array}{cc}
J_{1}^{\prime} P+P J_{1} & P J_{2} \\
J_{2}^{\prime} P & 0
\end{array}\right]\left[\begin{array}{l}
x \\
z
\end{array}\right]<0 \\
\forall J \in C o\left[\tilde{J}_{i}\right]_{i=1}^{q}, \forall\left[\begin{array}{l}
x \\
z
\end{array}\right]:\left[\begin{array}{ll}
J_{3} & J_{4}
\end{array}\right]\left[\begin{array}{l}
x \\
z
\end{array}\right]=0
\end{array}\right.
$$

Perceba que a definição 2 implica que $v(x)=x^{\prime} P x$ é função de Lyapunov para o sistema (2). Este fato pode ser verificado ao se eliminar a variável $z$ em (2), obtendose assim o sistema $\dot{x}=J_{0} x$ com $J_{0}=J_{1}-J_{2} J_{4}^{-1} J_{3} \mathrm{e}$ $\dot{v}(x)=x^{\prime}\left(J_{0}^{\prime} P+P J_{0}\right) x<0 \quad \forall x$, que é a condição usual de estabilidade quadrática.

O seguinte lema, conhecido como Lema de Finsler (Boyd et al., 1994), é a base dos resultados obtidos neste artigo.

Lema 1 Sejam $\Psi=\Psi^{\prime}$ e $C_{a}$ duas matrizes conhecidas. Então tem-se que

$$
X^{\prime} \Psi X<0 \text { onde } X \text { satisfaz } C_{a} X=0
$$


se e somente se $\exists L$ de dimensões compatíveis tal que

$$
\Psi+L C_{a}+C_{a}^{\prime} L^{\prime}<0
$$

É importante observar que o Lema 1 fornece condiço necessárias e suficientes apenas quando as matrizes $C_{a}$ ou $\Psi$ não apresentam parâmetros incertos. Quando $C_{a}$ ou $\Psi$ dependerem de um conjunto de parâmetros incertos, a condição de necessidade só continua válida se a matriz $L$ for também dependente deste mesmo conjunto de parâmetros incertos(Lu e Doyle, 1995).

O lema de Finsler acima se mostra particularmente interessante no tratamento de incertezas politópicas. O próximo lema, conhecido como "D-G scaling"é utilizado na última seção para o tratamento de incertezas na forma LFT.

Lema 2 Sejam $w_{i} \in R^{k}$ e $z_{i} \in R^{k}$. Existe um escalar $\delta_{i}$ tal que $w_{i}=\delta_{i} z_{i}$ e $\left|\delta_{i}\right| \leq 1$ se e somente se existem matrizes $S_{i}, T_{i}$ tais que

$$
\left\{\begin{array}{l}
w_{i}^{\prime} S_{i} w_{i} \leq z_{i}^{\prime} S_{i} z_{i}, \quad S_{i}>0 \\
w_{i}^{\prime} T_{i} z_{i}=0 T_{i}=-T_{i}^{\prime}, \quad T_{i}, S_{i} \in R^{n_{i} \times n_{i}}
\end{array}\right.
$$

A prova deste lema pode ser encontrada em Feron et al. (1996).

\section{SISTEMAS DE TEMPO CONTÍNUO}

O problema de análise da estabilidade quadrática de sistemas com restrições algébricas, sem eliminar a variável algébrica, é o enfoque desta seção.

A partir do Lema 1 e da Definição 2 apresenta-se a solução do problema de análise para o caso de sistemas nominais. Em seguida este problema é solucionado para sistemas com parâmetros incertos.

Teorema 1 O sistema

$$
\left\{\begin{array}{l}
\dot{x}=J_{1} x+J_{2} z \\
0=J_{3} x+J_{4} z
\end{array}\right.
$$

é assintoticamente estável e admissível se e somente se existem matrizes $P>0$ e $L$ tal que a seguinte LMI seja satisfeita:

$\left[\begin{array}{cc}J_{1}^{\prime} P+P J_{1} & P J_{2} \\ J_{2}^{\prime} P & 0\end{array}\right]+L\left[\begin{array}{ll}J_{3} & J_{4}\end{array}\right]+\left[\begin{array}{ll}J_{3} & J_{4}\end{array}\right]^{\prime} L^{\prime}<0$

Em caso afirmativo, $v(x)=x^{\prime} P x$ é uma função de Lyapunov para o sistema.
Prova: A estabilidade sai diretamente da aplicação do Lema 1 e da Definição 2. A partir de (9) e do Lema 1 tem-se que (9) será satisfeita se e somente se

$$
\begin{aligned}
& {\left[\begin{array}{l}
x \\
z
\end{array}\right]^{\prime}\left[\begin{array}{cc}
J_{1}^{\prime} P+P J_{1} & P J_{2} \\
J_{2}^{\prime} P & 0
\end{array}\right]\left[\begin{array}{l}
x \\
z
\end{array}\right]<0,} \\
& \forall\left[\begin{array}{l}
x \\
z
\end{array}\right]:\left[\begin{array}{ll}
J_{3} & J_{4}
\end{array}\right]\left[\begin{array}{l}
x \\
z
\end{array}\right]=0
\end{aligned}
$$

e o resultado segue pela Definição 2. Note que, se a condição (9) é satisfeita, então a matriz $J_{4}$ é inversível, provando assim a admissibilidade do sistema.

Este resultado é equivalente ao resultado dado por Takaba et al(1995) quando considera-se em (1) $E=$ $\left[\begin{array}{ll}I & 0 \\ 0 & 0\end{array}\right]$ e uma função de Lyapunov $v(\zeta)=\zeta^{\prime} E^{\prime} P \zeta$ com $E^{\prime} P=P E \geq 0$. O interessante aqui é que se obteve condições também necessárias e suficientes, com uma abordagem inicial diferente. Esta abordagem é bastante flexível e permite estender os resultados aqui apresentados em várias direções (veja Barbosa (1999)). Além disso as LMIs do teorema acima são estritas em contraste com a LMI $E^{\prime} P=P E \geq 0$ encontrada em Takaba et al(1995), que não possui solução estrita e é de difícil tratamento numérico pelos pacotes computacionais atualmente existentes para resolução de LMIs.

A seguir, considera-se o problema de análise da estabilidade para sistemas incertos.

Corolário 1 Seja $C o\left[\tilde{J}_{i}\right]_{i=1}^{q}$ um politopo convexo de vértices $\tilde{J}_{i}$ dados. Então o sistema incerto (2), (3) é quadraticamente estável e admissível se existem matrizes $P>0$ e L tal que a seguinte LMI seja satisfeita para todo $i=1, \ldots, q$

$$
\begin{aligned}
{\left[\begin{array}{cc}
J_{1 i}^{\prime} P+P J_{1 i} & P J_{2 i} \\
J_{2 i}^{\prime} P & 0
\end{array}\right]+} & L\left[\begin{array}{ll}
J_{3 i} & J_{4 i}
\end{array}\right] \\
& +\left[\begin{array}{ll}
J_{3 i} & J_{4 i}
\end{array}\right]^{\prime} L^{\prime}<0
\end{aligned}
$$

Em caso afirmativo, $v(x)=x^{\prime} P x$ é uma função de Lyapunov para o sistema (2), (3).

Prova: Supondo (10) satisfeita, então pela propriedade de convexidade, (10) tem que ser válida para todo $J \in$ $\operatorname{Co}\left[\tilde{J}_{i}\right]_{i=1}^{q}$, e neste caso a prova segue a prova do Teorema 1.

Observe que devido às condições do Lema 1 serem apenas suficientes quando têm-se matrizes incertas e L fixa, as condições do lema acima são apenas suficientes.

Considere o seguinte sistema incerto:

$$
\left\{\begin{array}{l}
\dot{x_{d}}=J_{1}^{\prime} x_{d}+J_{3}^{\prime} z_{d} \\
0=J_{2}^{\prime} x_{d}+J_{4}^{\prime} z_{d}
\end{array}, J_{d}=\left[\begin{array}{cc}
J_{1}^{\prime} & J_{3}^{\prime} \\
J_{2}^{\prime} & J_{4}^{\prime}
\end{array}\right]\right.
$$


onde $x_{d} \in R^{n}, z_{d} \in R^{l}$ são respectivamente os vetores de variáveis de estados e das variáveis algébricas.

Seja o sistema (11) denominado de Sistema Dual(D) e o sistema (2) de Sistema Primal(P). Note que o sistema (11) é obtido transpondo-se a matriz do sistema primal, ou seja, $J=J_{d}^{\prime}$. A mesma idéia se aplica ao eliminar a variável algébrica $z$ no sistema (2). Neste caso obtém-se $\dot{x}=J_{r} x$, onde $J_{r}=J_{1}-J_{2} J_{4}^{-1} J_{3}$, para o primal e $\dot{x_{d}}=$ $J_{r d} x_{d}$, onde $J_{r d}=J_{1}^{\prime}-J_{2}^{\prime} J_{4}^{\prime-1} J_{3}^{\prime}$, para o dual. Perceba que os autovalores da matriz $J_{r}$ são os mesmos de $J_{r d}$ e, portanto, a estabilidade do sistema (11) é equivalente à estabilidade do sistema (2).

Ressalta-se ainda que se $v(x)=x^{\prime} P x$ é uma função de Lyapunov para o sistema primal, então $v\left(x_{d}\right)=$ $x_{d}^{\prime} P^{-1} x_{d}$ é uma função de Lyapunov para o sistema dual e vice-versa. Este resultado continua sendo válido mesmo para certos tipos de funções de Lyapunov dependente dos parâmetros incertos (Trofino, 1998). A noção de sistema dual é interessante no caso de síntese, onde normalmente problemas de estabilização do sistema primal são mais facilmente resolvidos com a utilização do sistema dual.

Corolário 2 Seja $C o\left[\tilde{J}_{i}\right]_{i=1}^{q}$ um politopo convexo de vértices $\tilde{J}_{i}$ dados. Então o sistema incerto (2), (3) é quadraticamente estável e admissível se existem matrizes $W>0$ e L tal que para todo $i=1, \ldots, q$ :

$$
\begin{aligned}
{\left[\begin{array}{cc}
W J_{1 i}^{\prime}+J_{1 i} W & W J_{3 i}^{\prime} \\
J_{3 i} W & 0
\end{array}\right]+} & L\left[\begin{array}{cc}
J_{2 i}^{\prime} & J_{4 i}^{\prime}
\end{array}\right] \\
& +\left[\begin{array}{ll}
J_{2 i}^{\prime} & J_{4 i}^{\prime}
\end{array}\right]^{\prime} L^{\prime}<0
\end{aligned}
$$

Em caso afirmativo, $v(x)=x^{\prime} W^{-1} x$ é uma função de Lyapunov para o sistema (2), (3).

Prova: Suponha (12) satisfeita. Então por convexidade ela é válida $\forall J \in C o\left[\tilde{J}_{i}\right]_{i=1}^{q}$. Com o Teorema 1 temse que $v\left(x_{d}\right)=x_{d}^{\prime} W x_{d}$ é função de Lyapunov para o sistema dual, isto é, $J_{r d}^{\prime} W+W J_{r d}<0$. Logo para $P=W^{-1}: P J_{r d}^{\prime}+J_{r d} P=P J_{r}+J_{r}^{\prime} P<0$ e portando $v(x)=x^{\prime} P x$ é função de Lyapunov para o primal. $\diamond$

\section{SISTEMAS DE TEMPO DISCRETO}

No caso discreto, os sistemas com restrições algébricas são descritos por um conjunto de equações à diferenças (recursivas) e algébricas. Os resultados a seguir são uma extensão dos resultados obtidos para o caso contínuo.

Considere o sistema incerto

$$
\left\{\begin{array}{ccc}
x_{k+1} & = & J_{1} x_{k}+J_{2} z_{k} \\
0 & = & J_{3} x_{k}+J_{4} z_{k}
\end{array}, J=\left[\begin{array}{cc}
J_{1} & J_{2} \\
J_{3} & J_{4}
\end{array}\right]\right.
$$

onde $x_{k} \in R^{n}, z_{k} \in R^{l}$ são respectivamente os vetores das variáveis de estados e algébricas. Como no caso contínuo assume-se que as incertezas estão confinadas num politopo de vértices conhecidos como indicado a seguir:

$$
J \in C o\left[\tilde{J}_{i}\right]_{i=1}^{q}, \tilde{J}_{i}=\left[\begin{array}{ll}
J_{1 i} & J_{2 i} \\
J_{3 i} & J_{4 i}
\end{array}\right]
$$

De forma análoga ao caso contínuo, pode-se definir a noção de estabilidade quadrática para sistemas descritores a tempo discreto da seguinte forma:

Definição 3 (Estabilidade Quadrática) Considere o sistema (13),(14) e suponha que ele seja admissível. Então este sistema é quadraticamente estável se existe uma função $v\left(x_{k}\right)=x_{k}^{\prime} P x_{k}>0, \forall x_{k}$ tal que sua variação temporal para as trajetórias do sistema (2), (3) satisfaça:

$$
\left\{\begin{array}{l}
v\left(x_{k+1}\right)-v\left(x_{k}\right)= \\
{\left[\begin{array}{l}
x_{k} \\
z_{k}
\end{array}\right]^{\prime}\left(\left[\begin{array}{cc}
-P & 0 \\
0 & 0
\end{array}\right]+\left[\begin{array}{l}
J_{1}^{\prime} \\
J_{2}^{\prime}
\end{array}\right] P\left[\begin{array}{ll}
J_{1} & J_{2}
\end{array}\right]\right)\left[\begin{array}{l}
x_{k} \\
z_{k}
\end{array}\right]<0} \\
\forall J \in C o\left[\tilde{J}_{i}\right]_{i=1}^{q}, \forall\left[\begin{array}{l}
x_{k} \\
z_{k}
\end{array}\right]:\left[\begin{array}{ll}
J_{3} & J_{4}
\end{array}\right]\left[\begin{array}{l}
x_{k} \\
z_{k}
\end{array}\right]=0
\end{array}\right.
$$

Note que quando elimina-se a variável algébrica $z$ em (13), a definição 3 retorna a definição usual de estabilidade quadrática para sistemas discretos.

A seguir são apresentadas condições para a análise de estabilidade desta classe de sistemas.

Teorema 2 Seja $C o\left[\tilde{J}_{i}\right]_{i=1}^{q}$ um politopo convexo de vértices $\tilde{J}_{i}$ dados. Então, o sistema (13), (14) é quadraticamente estável e admissível se existem matrizes $P>0$ e L, tal que a seguinte LMI esteja satisfeita para todo $i=1, \ldots, q$

$$
\left.\begin{array}{c}
{\left[\begin{array}{cc}
-P & 0 \\
0 & 0
\end{array}\right]+L\left[\begin{array}{cc}
J_{3 i} & J_{4 i}
\end{array}\right]+\left[\begin{array}{c}
J_{3 i} \\
J_{4 i}
\end{array}\right] L^{\prime}} \\
P\left[\begin{array}{c}
J_{1 i}^{\prime} \\
J_{2 i}^{\prime}
\end{array}\right] P \\
-P
\end{array}\right]
$$

Em caso afirmativo, $v\left(x_{k}\right)=x_{k}^{\prime} P x_{k}$ é função de Lyapunov para o sistema (13),(14).

Prova: Considere que (2) esteja satisfeita. Então por convexidade, tem-se que ela é satisfeita $\forall J \in C o\left[\tilde{J}_{i}\right]_{i=1}^{q}$. Aplicando o Complemento de Schur na LMI (2) e em 
seguida o Lema 1 obtém-se:

$$
\begin{aligned}
& {\left[\begin{array}{l}
x_{k} \\
z_{k}
\end{array}\right]^{\prime}\left(\left[\begin{array}{cc}
-P & 0 \\
0 & 0
\end{array}\right]+\left[\begin{array}{l}
J_{1}^{\prime} \\
J_{2}^{\prime}
\end{array}\right] P\left[\begin{array}{ll}
J_{1} & J_{2}
\end{array}\right]\right)\left[\begin{array}{l}
x_{k} \\
z_{k}
\end{array}\right]} \\
& <0, \quad \forall\left[\begin{array}{l}
x_{k} \\
z_{k}
\end{array}\right]:\left[\begin{array}{ll}
J_{3} & J_{4}
\end{array}\right]\left[\begin{array}{l}
x_{k} \\
z_{k}
\end{array}\right]=0
\end{aligned}
$$

e a prova segue pela Definição 3 .

$\diamond$

A abordagem dual para sistemas a tempo discreto é análoga àquela considerada no caso contínuo. Dado o sistema primal (13) o seu correspondente sistema dual é dado por:

$$
\left\{\begin{array}{ccc}
\psi_{k+1} & = & J_{1}^{\prime} \psi_{k}+J_{3}^{\prime} \gamma_{k} \\
0 & = & J_{2}^{\prime} \psi_{k}+J_{4}^{\prime} \gamma_{k}
\end{array}, J_{d}=\left[\begin{array}{cc}
J_{1}^{\prime} & J_{3}^{\prime} \\
J_{2}^{\prime} & J_{4}^{\prime}
\end{array}\right]\right.
$$

onde $\psi_{k} \in R^{n}, \gamma_{k} \in R^{l}$ são respectivamente os vetores das variáveis de estados e algébricas.

Corolário 3 Seja $C o\left[\tilde{J}_{i}\right]_{i=1}^{q}$ um politopo convexo de vértices $\tilde{J}_{i}$ dados. Então o sistema incerto (13),(14) é quadraticamente estável e admissível se existem matrizes $W>0$ e L tal que a seguinte LMI esteja satisfeita para todo $i=1, \ldots, q$ :

$$
\left[\begin{array}{cc}
{\left[\begin{array}{cc}
-W & 0 \\
0 & 0
\end{array}\right]+L\left[\begin{array}{cc}
J_{2 i}^{\prime} & J_{4 i}^{\prime}
\end{array}\right]+\left[\begin{array}{c}
J_{2 i} \\
J_{4 i}
\end{array}\right] L^{\prime}} & {\left[\begin{array}{c}
J_{1 i} \\
J_{3 i}
\end{array}\right] W} \\
W\left[\begin{array}{ll}
J_{1 i}^{\prime} & J_{3 i}^{\prime}
\end{array}\right] & -W
\end{array}\right]
$$

$$
<0
$$

Em caso afirmativo $v\left(x_{k}\right)=x_{k}^{\prime} W^{-1} x_{k}$ é uma função de Lyapunov do sistema.

Prova: Será omitida, pois pode ser obtida de forma semelhante ao caso contínuo, utilizando a abordagem dual.

\section{EXTENSÃO PARA SISTEMAS LFT}

Os resultados obtidos na seção 3 são aqui transpostos para sistemas LFT ( Linear Fractional Transformations). Para mais detalhes sobre LFT veja, por exemplo, Doyle et al. (1991), Zhou et al. (1996). A representação de um sistema incerto por LFT pode ser visualizada na forma de um bloco com realimentação. Isso é indicado no diagrama de blocos da Figura 1, onde $G(s)=C(s I-A)^{-1} B+D$ representa a matriz de transferência do sistema.

Considere que $\Delta$ é uma matriz diagonal com elementos incertos, representada por

$$
\Delta \in \Delta_{s}=\left\{\Delta: \Delta=\operatorname{Diag}\left(\delta_{i} I_{n_{i}}\right), \quad \delta_{i} \in R,\left|\delta_{i}\right| \leq 1\right\}
$$

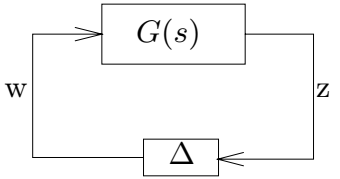

Figura 1: Sistema LFT

A representação por variáveis de estados do sistema na forma LFT é dada por

$$
\left\{\begin{array}{l}
\dot{x}=A x+B w \\
z=C x+D w \\
w=\Delta z
\end{array}\right.
$$

onde A, B, C, D são matrizes conhecidas e $x \in R^{n}$, $w \in R^{k}$ e $z \in R^{k}$.

Os teoremas a seguir se baseiam nos Lemas de Finsler e "D-G Scaling"apresentados na seção 2. O problema em questão é o de testar a estabilidade quadrática do sistema com $\Delta \in \Delta_{s}$. Para tal, assume-se que $\|D\|<1$, garantindo que o sistema (20) é regular, isto é, a matriz $\left(I-D \Delta_{i}\right)$ é inversível $\forall \Delta \in \Delta_{s}$.

Teorema 3 Seja $\left\{\Delta_{i}\right\}_{i=1}^{q}$ o conjunto de vértices do politopo $\Delta_{s}$. Então o sistema incerto (20) com $\Delta \in \Delta_{s} e$ $\|D\|<1$ é quadraticamente estável se existem matrizes $L$ e $P>0$, tal que para todo $i=1, \ldots, q$

$$
\begin{gathered}
{\left[\begin{array}{cc}
A^{\prime} P+P A & P B \Delta_{i} \\
\Delta_{i} B^{\prime} P & 0
\end{array}\right]+L\left[\begin{array}{ll}
C & \left(D \Delta_{i}-I\right)
\end{array}\right]} \\
+\left[\begin{array}{ll}
C & \left(D \Delta_{i}-I\right)
\end{array}\right]^{\prime} L^{\prime}<0
\end{gathered}
$$

Em caso afirmativo, $v(x)=x^{\prime} P x$ é função de Lyapunov para o sistema.

Prova: Com a eliminação da variável $w$, o sistema (20) é equivalente ao seguinte sistema na forma algébricodiferencial desacoplado:

$$
\left\{\begin{array}{l}
\dot{x}=A x+B \Delta z \\
0=C x+(D \Delta-I) z
\end{array}\right.
$$

Considerando $J_{1}=A, J_{2}=B \Delta, J_{3}=C$ e $J_{4}=(D \Delta-$ $I)$, pode-se facilmente aplicar o Teorema 1 no sistema (20) completando a prova.

Perceba que a condição $\|D\|<1$ é necessária para garantir a regularidade do sistema, isto é, $J_{4}$ inversível para todo $\Delta$. O interessante da abordagem acima é que se obteve uma formulação politópica para as incertezas do tipo LFT que são tipicamente tratadas como limitadas em norma através do uso do "D-G scaling". 
O resultado abaixo segue esta linha e é similar ao resultado obtido na Proposição 1.1 de Feron et al. (1996).

Teorema 4 Seja $\Delta_{s}$ definido por (19). Então o sistema incerto (20) com $\Delta \in \Delta_{s} e\|D\|<1$ é quadraticamente estável se existem matrizes $T, S$ e $P$, tais que:

$$
\begin{aligned}
& {\left[\begin{array}{cc}
A^{\prime} P+P A+C^{\prime} S C & P B+C^{\prime} S D+C^{\prime} T^{\prime} \\
B^{\prime} P+D^{\prime} S C+T C & -S+D^{\prime} S D+T D+D^{\prime} T^{\prime}
\end{array}\right]<0} \\
& T=-T^{\prime}, \quad T=\operatorname{Diag}\left\{T_{i}\right\}, \quad T_{i} \in R^{n_{i} \times n_{i}} \\
& S>0, \quad S=\operatorname{Diag}\left\{S_{i}\right\}, \quad S_{i} \in R^{n_{i} \times n_{i}}, \quad P>0
\end{aligned}
$$

Em caso afirmativo, $v(x)=x^{\prime} P x$ é função de Lyapunov para o sistema.

Prova: A prova segue os mesmos passos de Feron et al. (1996) na prova da Proposição 1.1 e será aqui omitida. $\diamond$

Note que os dois teoremas precedentes propõem condições diferentes para tratar o mesmo conjunto de incertezas. No primeiro, as incertezas são tratadas como politópicas através do Lema 2.1 (Finsler) e no segundo como limitadas em norma através do Lema 2.2 (D-G scaling). Tipicamente, as condições do Teorema 3 são menos restritivas que as do Teorema 4. No entanto, na abordagem politópica o número de restrições (LMIs) cresce exponencialmente com o número de parâmetros incertos, o que não ocorre na abordagem que utiliza o "D-G scaling". A seguir propõe-se um resultado que combina as duas abordagens acima.

Teorema 5 Seja $\Delta_{s}$ definido em (19). O sistema incerto (20) com $\Delta \in \Delta_{s}$ e $\|D\|<1$ é quadraticamente estável se existem matrizes $L, P, S$ e $T$, tais que:

$$
\begin{aligned}
& {\left[\begin{array}{ccc}
A^{\prime} P+P A & P B & 0 \\
B^{\prime} P & -S & T \\
0 & T^{\prime} & S
\end{array}\right]+L\left[\begin{array}{lll}
C & D & -I
\end{array}\right]+\left[\begin{array}{c}
C \\
D \\
-I
\end{array}\right] L^{\prime}} \\
& <0 \\
& T=-T^{\prime}, \quad T=\operatorname{Diag}\left\{T_{i}\right\} \\
& S>0, \quad S=\operatorname{Diag}\left\{S_{i}\right\}, \quad P>0
\end{aligned}
$$

Em caso afirmativo, $v(x)=x^{\prime} P x$ é função de Lyapunov para o sistema.

Prova: As condições para que $v(x)=x^{\prime} P x$ seja uma função de Lyapunov para o sistema (20) são $P>0$ e

$$
\dot{v}(x)=x^{\prime}\left(A^{\prime} P+P A\right) x+w^{\prime} B^{\prime} P x+x^{\prime} P B w<0
$$

sempre que $0=C x+D w-z$ e $w=\Delta z$.
Como no Teorema 4, pode-se tratar a condição $w=\Delta z$ com o "D-G scaling", resultando em

$$
\dot{v}(x) \leq\left[\begin{array}{c}
x \\
w \\
z
\end{array}\right]\left[\begin{array}{ccc}
A^{\prime} P+P A & P B & 0 \\
B^{\prime} P & -S & T \\
0 & T^{\prime} & S
\end{array}\right]\left[\begin{array}{c}
x \\
w \\
z
\end{array}\right] \leq 0
$$

sempre que $\left[\begin{array}{ccc}C & D & -I\end{array}\right]\left[\begin{array}{c}x \\ w \\ z\end{array}\right]=0$. A condição (24) é então suficiente para a estabilidade quadrática do sistema.

Supondo que (22) esteja satisfeita, pode-se simplesmente pré e pós multiplicá-la por $X=\left[\begin{array}{lll}x^{\prime} & w^{\prime} & z^{\prime}\end{array}\right]$ e $X^{\prime}$ respectivamente e o teorema fica provado com (24). $\diamond \diamond$

A vantagem das abordagens dos dois últimos teoremas é o fato do número de LMIs ser independente do número de parâmetros incertos no sistema. Como mencionado anteriormente, o número de LMIs cresce exponencialmente com o número de parâmetros incertos.

Proposição 1 Se o Teorema 5 for satisfeito, o Teorema 4 também estará com

$$
L_{1}=\frac{1}{2} C^{\prime} S, L_{2}=T+\frac{D^{\prime} S}{2} \text { e } L_{3}=S\left(\frac{1}{2}+\epsilon\right)
$$

sendo $\epsilon>0$ um escalar suficientemente pequeno.

Prova: Considere as condições dadas pelo Teorema 5 . Defina $L=\left[\begin{array}{lll}L_{1}^{\prime} & L_{2}^{\prime} & L_{3}^{\prime}\end{array}\right]^{\prime}$. Então a LMI (23) fica:

$$
\left[\begin{array}{ccc}
\Gamma & P B+L_{1} D+C^{\prime} L_{2}^{\prime} & -L_{1}+C^{\prime} L_{3}^{\prime} \\
* & -S+L_{2} D+D^{\prime} L_{2}^{\prime} & T-L_{2}+D^{\prime} L_{3}^{\prime} \\
* & * & S-L_{3}-L_{3}^{\prime}
\end{array}\right]<0
$$

com $\Gamma=A^{\prime} P+P A+L_{1} C+C^{\prime} L_{1}^{\prime}$. Escolhendo $L_{1}=$ $\frac{1}{2} C^{\prime} S$ e $L_{2}=T+\frac{D^{\prime} S}{2}$ e $L_{3}=S\left(\frac{1}{2}+\epsilon\right)$ e definindo

$$
\Phi=\left[\begin{array}{cc}
A^{\prime} P+P A+C^{\prime} S C & P B+C^{\prime} S D \frac{1}{2}+C^{\prime}\left(T+\frac{D^{\prime} S}{2}\right) \\
* & -S+T D+D^{\prime} T^{\prime}+D^{\prime} S D
\end{array}\right]
$$

tem-se que:

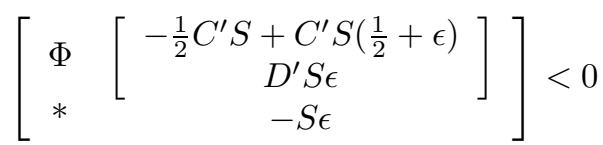

Aplicando Schur obtém-se

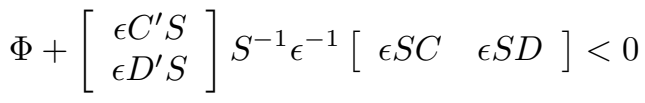

que poder ser reescrita na forma

$$
\Phi+\epsilon\left[\begin{array}{l}
C^{\prime} \\
D^{\prime}
\end{array}\right] S\left[\begin{array}{ll}
C & D
\end{array}\right]<0
$$

Para $\epsilon$ suficientemente pequeno, a expressão acima se reduz à condição dada pelo Teorema 4 completando a prova. 


\section{EXEMPLO NUMÉRICO}

Nos exemplos a seguir utiliza-se o Pacote Computacional Scilab para resolução de LMIs. Este pacote está disponível no site: www-rocq.inria.fr/scilab.

Exemplo 1 Considere o problema de análise da estabilidade para um sistema com incertezas nas matrizes $J_{3}$ e $J_{4}$, descrito por (2) com:

$$
\begin{gathered}
J_{1}=\left(\begin{array}{cc}
-10 & -5 \\
-14 & -11
\end{array}\right), \quad J_{2}=\left(\begin{array}{ll}
2 & 0 \\
7 & 3
\end{array}\right) \\
J_{3}=\left(\begin{array}{cc}
6 & 8 \\
6 & 6\left(1+\delta_{1}\right)
\end{array}\right), \quad J_{4}=\left(\begin{array}{cc}
8 & 5 \\
6 & 6\left(1+\delta_{2}\right)
\end{array}\right)
\end{gathered}
$$

Note que o sistema nominal é estável, dado que os autovalores de $\left(J_{1}-J_{2} J_{4}^{-1} J_{3}\right)$ quando $\delta_{1}=0$ e $\delta_{2}=0$ são -2.4262859 e -26.240381 .

Aplicando o Teorema 1, consegue-se provar que o sistema acima continua quadraticamente estável para uma variação de $70 \%$ em $\delta_{1}$ e de $80 \%$ em $\delta_{2}$.

Exemplo 2 Para exemplificar os resultados obtidos na seção 5 , considere o sistema ( 20) com:

$$
\begin{gathered}
A=\left(\begin{array}{cccc}
0 & 1 & 0 & 0 \\
0 & 0 & 1 & 0 \\
0 & 0 & 0 & 1 \\
-1 & -4 & -6 & -4
\end{array}\right), B=\left(\begin{array}{cc}
0 & 0 \\
0 & 0 \\
0 & 0 \\
1 & -1
\end{array}\right) \\
C=\left(\begin{array}{llll}
0 & 7 & 0 & 0 \\
6 & 1 & 2 & 2
\end{array}\right), D=\left(\begin{array}{cc}
0.1 & 0 \\
0 & 0.2
\end{array}\right)
\end{gathered}
$$

O sistema tem dois parâmetros incertos, $\delta_{1}$ e $\delta_{2}$, descritos por (19), que são limitados por $\left|\delta_{1}\right| \leq \alpha_{1}$ e $\left|\delta_{2}\right| \leq \alpha_{2}$. O objetivo é determinar o limite máximo possível de $\alpha_{1}$ e $\alpha_{2}$, de modo a garantir a estabilidade do sistema, utilizando-se os teoremas da seção 5. Para tal, fixa-se o limitante de um dos parâmetros e busca-se o maior limitante possível para o outro. Na Tabela 1 tem-se os valores obtidos para quatro diferentes situações, nos casos 1 e 2 fixa-se o valor de $\alpha_{2}$ em 0 e 0.14 , respectivamente, e nos casos 3 e 4 fixa-se o valor de $\alpha_{1}$ em 0.15 e 0 .

\begin{tabular}{|c|c|c||c|c|c|c|c|}
\cline { 3 - 5 } \multicolumn{1}{c|}{} & \multicolumn{2}{c|}{ Teorema 3} & \multicolumn{2}{c|}{ Teorema 4} & \multicolumn{2}{c|}{ Teorema 5} \\
\hline Casos & $\alpha_{1}$ & $\alpha_{2}$ & $\alpha_{1}$ & $\alpha_{2}$ & $\alpha_{1}$ & $\alpha_{2}$ \\
\hline \hline 1 & 0.45 & 0.0 & 0.42 & 0.0 & 0.42 & 0.0 \\
\hline 2 & 0.33 & 0.14 & 0.17 & 0.14 & 0.18 & 0.14 \\
\hline 3 & 0.15 & 0.20 & 0.15 & 0.14 & 0.15 & 0.15 \\
\hline 4 & 0.0 & 0.24 & 0.0 & 0.17 & 0.0 & 0.17 \\
\hline
\end{tabular}

Tabela 1: valores obtidos para $\alpha_{i}$ em quatro situações

Com os resultados da Tabela 1, pode-se confirmar que a abordagem politópica (Teorema 3 ) é menos restritiva que as outras abordagens. Já foi provado analiticamente na Proposição 5.1 que, se o Teorema 5 estiver satisfeito o Teorema 4 também estará. No entanto, com os resultados numéricos obtidos, não foi possível concluir se o Teorema 5 é ou não mais abrangente que o Teorema 4. Este ponto ficará em aberto como tema para futuras pesquisas.

\section{CONCLUSÕES}

Neste artigo foram apresentadas condições suficientes para a estabilidade quadrática de sistemas incertos de tempo contínuo e discreto com restrições algébricas no estado. As condições exploram a estrutura original do sistema, permitindo que os resultados possam ser estendidos em várias direções (Barbosa, 1999; Barbosa e Trofino, 2000b; Barbosa e Trofino, 2000a).

Outro ponto interessante desta abordagem é que se pode de maneira simplificada considerar incertezas em todos os elementos do sistema. Além disso, as incertezas podem ser tratadas como politópicas ou como limitadas em norma. Esse ponto é discutido na seção 5 com a apresentação de três teoremas e o estabelecimento de algumas relações entre estes resultados.

\section{AGRADECIMENTOS}

Os autores agradecem o Conselho Nacional de Desenvolvimento Científico e Tecnológico - CNPq, pelo suporte financeiro, durante a realização deste trabalho.

\section{REFERÊNCIAS}

Barbosa, K. A. (1999). Técnicas LMI para Análise e Sintese de Sistema com Restrições Algébricas no Estado, Master's thesis, Pós Graduação em Engenharia Elétrica - UFSC, Fpolis - SC.

Barbosa, K. A. e Trofino, A. (2000a). Síntese $H_{2}$ para Sistemas com Restrições Algébricas , XIII Congresso Brasileiro de Automática, Florianópolis,SC.

Barbosa, K. A. e Trofino, A. (2000b). Síntese $H_{\infty}$ para Sistemas com Restrições Algébricas no Estado, XIII Congresso Brasileiro de Automática, Florianópolis,SC.

Boyd, S., Ghaoui, L., Feron, E. e Balakrishnan, V. (1994). Linear Matrix Inequalities in System and Control Theory, SIAM Studies in Applied Mathematics. 
Cobb, D. J. (1984). Feedback and pole assignment in descriptor systems, Int. Journal Control Vol. 33: $1135-1146$.

Dai, L. (1989). Singular Control System, SpringVerlag.

Doyle, J. C., Packard, A. e Zhou, K. (1991). Review of LFT, LMIs, and $\mu$, IEE-Proceedings of the 30th Conference on Decision and Control, pp. 12271232 .

Feron, E., Apkarian, P. e Gahinet, P. (1996). Analysis and Synthesis of Robust Control Systems via Parameter-Dependent Lyapunov Functions, IEEE Transactions on Automatic Control Vol. 41(No 7): 1041-1046.

Lu, W.-M. e Doyle, J. (1995). $H_{\infty}$ control of nonlinear systems: a convex characteriztion, IEEE Transactions on Automatic Control Vol. 40(No 9): 16681675 .

Takaba, K., Morihira, N. e Katayama, T. (1995). A generalized Lyapunov theorem for descriptor system, Systems 83 Control Letters Vol. 24: 49-51.

Trofino, A. (1998). Abordagem LMI para problemas de controle com funções de Lyapunov dependente de parâmetros, XII Congresso Brasileiro de Automática, Uberlânica, MG. mini-curso.

Varga, A. (1995). On stabilization methods of descriptor systems, Systems and Control Vol. 24: 133138.

Verghese, G., Lévi, B. C. e Kailath, T. (1981). A Generalized State-Space for Singular Systems, IEEE Transactions on Automatic Control Vol. 26(No 4): 811831.

Zhou, K., Doyle, J. C. e Glover, K. (1996). Robust and Optimal Control, Prentice-Hall International, Inc., USA. 